Frauenwelten in der Antike 


\section{Frauenwelten in der Antike}

\section{Geschlechterordnung}

und weibliche Lebenspraxis

Mit 162 Quellentexten und Bildquellen

Herausgegeben von Thomas Späth

und Beate Wagner-Hasel

Verlag J. B. Metzler Stuttgart $\cdot$ Weimar 
Die Deutsche Bibliothek - CIP-Einheitsaufnahme

Frauenwelten in der Antike: Geschlechterordnung und weibliche Lebenspraxis ; mit Quellentexten und Bildquellen / hrsg. von Thomas Späth und Beate Wagner-Hasel. - Stuttgart ; Weimar : Metzler, 2000

ISBN 978-3-476-01677-5

ISBN 978-3-476-01677-5

ISBN 978-3-476-03779-4 (eBook)

DOI 10.1007/978-3-476-03779-4

Dieses Werk einschließlich aller seiner Teile ist urheberrechtlich geschützt. Jede Verwertung außerhalb der engen Grenzen des Urheberrechtsgesetzes ist ohne Zustimmung des Verlages unzulässig und strafbar. Das gilt insbesondere für Vervielfältigungen, Übersetzungen, Mikroverfilmungen und die Einspeicherung und Verarbeitung in elektronischen Systemen.

(C) 2000 Springer-Verlag GmbH Deutschland

Ursprünglich erschienen bei J.B.Metzlersche Verlagsbuchhandlung und Carl Ernst Poeschel Verlag GmbH in Stuttgart 2000

www.metzlerverlag.de info@metzlerverlag.de 


\section{Inhalt}

Hinweise zur Benutzung des Buches . . . . . . . . . . . . VII

Einleitung ........................ IX

I. Heirat und Zugehörigkeit . . . . . . . . . . . . . . . . . . . . . 1

Haus und Schleier in Mesopotamien (Brigitte Groneberg) . . . . . . . . . . 1

Heirat und Bürgerstatus in Athen (Elke Hartmann) . . . . . . . . . . . . . . 16

Eheallianzen und Ehealltag in Rom (Christiane Kunst) . . . . . . . . . . . 32

II. Kultpraktiken . . . . . . . . . . . . . . . . . . 53

Kulträume von Frauen in Athen: Das Beispiel der Artemis Brauronia

(Katharina Waldner) . . . . . . . . . . . . . . . . . . . . . . . . 53

Die Reglementierung von Traueraufwand und die Tradierung des Nachruhms der Toten in Griechenland (Beate Wagner-Hasel) . . . . . . . . . . . 81

Klagende Frauen. Zur weiblichen Trauerhaltung in Rom

(Francesca Prescendi) . . . . . . . . . . . . . . . . . . . . . . . . . . . . . 102

Die vestalischen Jungfrauen (Hildegard Cancik-Lindemaier) . . . . . . . . . . 111

Matralia und Matronalia: Feste von Frauen in der römischen Religion

(Francesca Prescendi) . . . . . . . . . . . . . . . . . . . . . . . . . . . . . 123

III. Wissen und Tradition $\ldots \ldots \ldots$

Weissagung und Macht: Die Pythia (Christine Schnurr-Redford) . . . . . . . 132

Eine römische Dichterin: Sulpicia (Christine Rohweder) . . . . . . . . . . . 147

Philosophinnen (Henriette Harich-Schwarzbauer) . . . . . . . . . . . . . . . 162

Perpetua: Visionen im Christentum (Peter Habermehl) . . . . . . . . . . . 174

Die Aristokratin als Mäzenin und Autorin im Byzanz der Komnenenzeit

(Ruth E. Harder) . . . . . . . . . . . . . . . . . . 183

IV. Macht und Politik . . . . . . . . . . . . . . . . . . . . . 198

Das Diktum der Philosophen: Der Ausschluss der Frauen aus der Politik und die Sorge vor der Frauenherrschaft (Beate Wagner-Hasel) . . . . . . . . 198

Weiblichkeitsideale in der römischen Welt: Lucretia und die Anfänge der Republik (Francesca Prescendi) . . . . . . . . . . . . . . . . . . . . . . 217

Sexuelle Diffamierung und politische Intrigen in der Republik: P. Clodius

Pulcher und Clodia (Rosmarie Günther) . . . . . . . . . . . . . . . . . . . 227

Das Idealbild der römischen Kaiserin: Livia Augusta (Angelika Dierichs) . . . 241 
Skrupellose Herrscherin? Das Bild der Agrippina minor bei Tacitus

(Thomas Späth) . . . . . . . . . . . . . . . . . . . . . . . 262

Die Augusta aus der Wüste - die palmyrenische Herrscherin Zenobia (Anja Wieber) . . . . . . . . . . . . . . . . . . . . . . . . . . . . . . . . 281

V. Arbeitswelt und weibliche Geselligkeit . . . . . . . . . . . . . . . . 311

Arbeit und Kommunikation (Beate Wagner-Hasel) . . . . . . . . . . . . . . 311

Vermitteln, Verkuppeln und soziales Spiel. Informelle Geschäftstätigkeit von Frauen in hellenistischer Zeit (Wolfgang Christian Schneider) . . . . . . . 335

Matrone, vilica und ornatrix. Frauenarbeit in Rom zwischen Topos

und Alltagswirklichkeit (Rosmarie Günther) . . . . . . . . . . . . . . . . . 350

VI. Erotik und Sexualität _ . . . . . . . . . . . . . . . . . . . . . . 377

Hetären im klassischen Athen (Elke Hartmann) . . . . . . . . . . . . . . . 377

Erotik in der Bildenden Kunst der Römischen Welt (Angelika Dierichs) . . . 394

Das Ende der antiken Leiblichkeit. Begehren und Enthaltsamkeit bei

Ambrosius, Augustin und Maximian (Wolfgang Christian Schneider) . . . . . 412

Hieronymus und Paula: Briefe an eine Asketin und Mutter (Diemut Zittel) 426

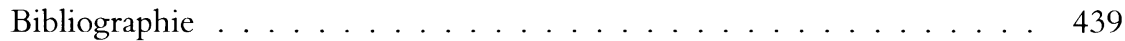

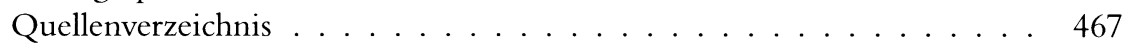

Autorinnen und Autoren des Bandes . . . . . . . . . . . . . . . . . . . . 477

Sach- und Namenregister . . . . . . . . . . . . . . . . . . . . . . 481

Stellenregister . . . . . . . . . . . . . . . . . . 487 


\section{Hinweise zur Benutzung des Buches}

Den Bild- und Textquellen in den einzelnen Beiträge sind nur kurze Hinweise auf die entsprechende Textstelle oder das Bildmotiv beigefügt; die ausführlichen Referenzen zu den benutzten Übersetzungen und Texteditionen sowie die Abbildungsnachweise finden sich im ausführlichen Quellenverzeichnis im Anhang (S. 467) zusammengestellt.

In Text und Anmerkungen werden moderne Publikationen abgekürzt zitiert mit Namen der Autorinnen und Autoren, Kurztitel und Erscheinungsjahr der benutzten Ausgabe. Die vollständigen bibliographischen Angaben enthält die Bibliographie am Ende des Bandes.

Antike AutorInnen werden mit vollständigem Namen zitiert; die Abkürzungen der Werke folgt dem Abkürzungsverzeichnis des Kleinen Pauly, gegebenenfalls dem Liddell-Scott, respektive dem Oxford Latin Dictionary.

Die einzelnen Beiträgen schliessen mit Hinweisen auf "weitere Quellen« sowie auf "grundlegende" und "weiterführende» Literatur. Unter der "grundlegenden Literatur" sind im allgemeinen Publikationen aufgefuhrt, die zum spezifischen Thema des Beitrags und den präsentierten Quellen ergänzende oder vertiefende Informationen bieten; "weiterführende Literatur" stellt Publikationen zusammen, die über die Fragestellung des Beitrags und der Quellen hinaus einen Zugang zu benachbarten Themen eröffnen. 


\section{Einleitung}

\section{Neue Fragen an ein altes Thema: Frauen- und Geschlechtergeschichte in den Altertumswissenschaften}

Beate Wagner-Hasel und Thomas Späth

"Ich sehe nicht ein, warum wir uns immer um die Männer oder gar um ihre Schlachten kümmern sollten; die Geschichte der Frauen ist meist viel interessanter." Dieses Zitat aus der Feder Theodor Fontanes ziert den Umschlag einer jüngsten Studie über die Geschichte der europäischen Frauen aus der Perspektive einer Neuzeithistorikerin. ${ }^{1}$ Wie bereits andere Historikerinnen vor ihr, die in den letzten zwanzig Jahren ein neues Kapitel der Frauenforschung aufschlugen, ${ }^{2}$ erinnert Gisela Bock an jene Phase der Geschichtsforschung des 19. Jh., als Geschichte die Geschichte von Nationalstaaten und der von Männern bestrittenen Schlachten war und für Frauen keine Identifikationsangebote enthielt. Der Verweis dient der Selbstvergewisserung einer Tradition, die die Erforschung der Geschichte der Frauen als etwas Neues erscheinen lässt. Aber eben dies ist Frauengeschichte nur bedingt.

Wenn antike Historiographen ihre Schlachtenbeschreibungen verfassten, sparten sie das Schicksal von Frauen keineswegs immer aus. In seinem Plädoyer für eine 'pragmatische` Geschichtsschreibung kritisiert der Geschichtsschreiber Polybios im 2. Jh. v. Chr. seinen Kollegen Phylarchos: "In dem Bemühen [...], die Leser durch seine Erzählung zum Mitleid zu stimmen und tiefes Erbarmen in ihnen zu wecken, wartet er mit Umarmungen der Frauen, Ausraufen der Haare, Entblößen der Brüste auf, dazu mit Tränen und Wehklagen von Männern und Frauen, die zusammen mit ihren Kindern und den alten Eltern fortgeführt wurden. Und so verfährt er in seinem ganzen Geschichtswerk, immer und überall sucht er uns Greuel vor Augen zu stellen. Wir wollen das Unwürdige und Weibische solcher Effekthascherei auf sich beruhen lassen und nur fragen, ob dergleichen der Aufgabe eines Ge-

1 Воск, Geschichte der europäischen Frauen 2000.

2 Vgl. etwa Gianna Poмata, die an den Anfang ihrer Untersuchung Die Geschichte der Frauen zwischen Anthropologie und Biologie von 1983 (eine Teilübersetzung von La storia delle donne: una questione di confine 1983, publiziert in: Feministische Studien 2/2) einen Ausspruch der Heldin des Romans Northanger Abbey von Jane Austen (1818) über ihre Lektüre von Geschichtswerken stellte: „Auf jeder Seite Streit zwischen Päpsten und Kaisern, Kriege und Seuchen. Die Männer sind im allgemeinen Gauner, und die Frauen - Frauen gibt es darin praktisch nie; eine grässliche Langeweile“ (113). 
schichtswerkes entspricht oder ihr dient. « ${ }^{3}$ Die ablehnende Haltung des Polybios gegenüber dem Vorgehen eines Phylarchos oder auch eines Duris von Samos ${ }^{4}$ kann nicht darüber hinwegtäuschen, dass antike Geschichtsschreibung sich keineswegs nur in nüchternen Schlachtenbeschreibungen zum Nutzen späterer Kriegsstrategen erschöpfte. In den Historien Herodots, die von den Perserkriegen handeln, finden wir nicht nur lange ethnographische Exkurse über die Lebensweise von Männern und Frauen, sondern auch Informationen über militärische Aktionen einer Pheretime, die in Kyrene die Herrschaft erlangte. ${ }^{5}$ Und die Chronisten der Kriegszüge Alexander des Großen nahmen in ihre Schilderungen stets auch die Begegnung Alexanders mit kriegerischen Frauen, den Amazonen, auf. ${ }^{6}$

Frauen waren aus dem historiographischen Gedächtnis der Alten nicht verbannt und gelehrte Frauen wie Christine de Pizan konnten in der frühen Neuzeit für die Gestaltung ihrer Stadt der Frauen auf einen reichen Fundus antiker Beispiele zurückgreifen. ${ }^{7}$ Aus Plutarchs Schrift Von der Tugend der Frauen aus dem 1./2. Jh. n. Chr. schöpften die Gründungsväter der amerikanischen Verfassung Beispiele für sstaatstragendes Verhalten von Frauen, um die Bürgerinnen ihres Landes auf die neue Republik einzuschwören. ${ }^{8}$ In den Universalgeschichten des 18. Jh., die nicht nur von Schlachten und Kriegen, sondern von den Sitten und Gebräuchen, von Kleidung, Hausrat, Wirtschaft, Prunk und Benehmen handelten, waren Männer wie Frauen der Antike gleichermaßen präsent. ${ }^{9}$ Auch wenn sich die althistorische Zunft im 19. Jh. zunehmend einer auf Staat und Politik ausgerichteten Geschichtsbetrachtung verschrieb und vielen älteren Werken die Würde "historischer Wissenschaftlichkeit " absprach, so entfernte sie sich doch nicht völlig von der Tradition der Universalgeschichte. ${ }^{10}$ Selbst ein Altertumswissenschaftler wie Theodor Mommsen, der wesentlich zur ,Verwissenschaftlichung« der Geschichtsschreibung beitrug, mochte nicht immer auf effektvolle und farbige Darstellung der Vergan-

3 Polybios 2,56,7-9; Übers. nach Hans Drexler (Polybios, Geschichte. Gesamtausgabe in zwei Bänden. Zürich/Stuttgart 1963).

4 Polybios 2,56,10: „Der Historiker soll seine Leser nicht durch Schauergeschichten in Erschütterung versetzen, keine schönen Reden einlegen, die vielleicht so hätten gehalten werden können, nicht das Geschehen mit Nebenzügen und Begleitumständen schmücken, wie es die Tragödiendichter tun, einzig und allein das wirklich Getane und Gesagte berichten, auch wenn es nur schlichte Dinge sind " (In 2,56,11-12 begründet Polybios seine Ansicht über die gegensätzliche Zielsetzungen von Tragödie und Geschichtsschreibung). Zur Debatte um die sogenannte spragmatischer und stragischer Geschichtsschreibung vgl. zusammenfassend und mit Literaturhinweisen SpÄTH, Salluste 1998, $176 \mathrm{ff}$.

5 Herodot 4,162-167.

6 Vgl. etwa Pompeius Trogus bei Justinus 2,4,30; Quintus Curtius Rufus, Von den Taten Alexanders des Großen 6,19; Arrian, Anabasis 7,6.

7 DE Pizan, Das Buch von der Stadt der Frauen 1990, $72 \mathrm{f}$.

8 Richard, The Founders and the Classics 1994, 53.

9 Vgl. Stollberg-Rilinger, Väter der Frauengeschichte? 1996, 39-71.

10 Zum Wechsel von der Universal- zur Nationalgeschichte Nippel, Griechen, Barbaren und Wilder. 1990, insb. 78; zum spannungsreichen Verhältnis zwischen breiten rantiquarischen und wissenschaftlich historischen Interessen vgl. Momigliano, Ancient History and the Antiquarian 1950) (deutsche Übers. 1999). 
genheit verzichten. In seinem Werk Römische Geschichte, für das er 1902 den Nobelpreis für Literatur erhielt, schildert er beispielsweise mit warmer Einfühlung das häusliche Regiment des gestrengen römischen Feldherrn Cato und dessen Kritik am weiblichen Luxusstreben. ${ }^{11}$ Die Darstellung des Frauenlebens in Rom spielt in Mommsens Werk zwar nur eine untergeordnete Rolle, aber im 19. Jh. entstanden parallel zu den neuen Geschichten der griechischen oder römischen `Nation`zahllose Einzelstudien zu Themen, die Frauen betrafen oder ihrer Stellung in antiken Gesellschaften ganz allgemein gewidmet waren: Steht Carl Gotthold Lenz' Geschichte der Weiber im heroischen Zeitalter von 1790 mit seiner stark auf kulturelle Praktiken ausgerichteten Betrachtung noch in der Tradition der Universalgeschichten des 18. Jh., so zeigen Schriften wie Ivo Bruns' Frauenemanzipation im Altertum von 1905 ein verändertes Gesicht der Geschichtswissenschaft. Die großen sozialen und politischen Fragen der Zeit, die Kämpfe um rechtliche und politische Gleichstellung von Frauen, haben sich gerade zu Beginn des 20.Jh. in neuen Fragestellungen und Themenorientierungen der Altertumswissenschaften niedergeschlagen und zur Entstehung von rechts- und sozialgeschichtlichen Studien geführt. Neben den fachwissenschaftlichen Untersuchungen zur Geschäftsfähigkeit der griechischen Frau von Alexander Balabanoff (Leipzig 1905) oder zur Politischen Wirksamkeit der griechischen Frau von Otto Braunstein (Leipzig 1911) gab es im 19. und frühen 20. Jh. eine Reihe von historischen Werken aus der Feder von Amateuren. Es waren dies oft Frauen, die ihre Bildung außerhalb der universitären Institutionen erhalten hatten. Aus Lily Brauns Darstellung der Rolle der Frauen im Altertum in Die Frauenfrage. Ihre geschichtliche Entwicklung und ihre wirtschaftliche Seite (Leipzig 1901, frz. 1908: Le problème de la femme, son évolution historique, son aspect économique), in der diese den Mythos vom Matriarchat angriff, hat noch Simone de Beauvoir in ihrer Darstellung Le detixième sexe/Das andere Geschlecht (1949/1951) geschöpft; Bertha Eckstein-Dieners Mütter und Amazonen von 1927 bildete das erste Kultbuch der Frauenbewegung der 70er Jahre, die sich ihrer eigenen Geschichte vergewissern wollte. ${ }^{12}$

Die Beschäftigung mit den Frauen in der Antike ist nicht neu; neu sind jeweils nur die Fragestellungen und die Methoden, mit denen an die antiken Befunde herangegangen wird. Neu ist gewiss auch das Ausmaß der Beschäftigung mit ihnen. Die Forschungen zu Frauen in der Antike sind mittlerweile so zahlreich geworden, dass es schwierig ist, einen Überblick über die Vielfalt der Neuerscheinungen zu gewinnen. ${ }^{13}$ Galten die Publikationen der 70er und frühen 80er Jahre noch pau-

11 Theodor Mommsen, Römische Geschichte: Drittes Buch: Von der Einigung Italiens bis auf die Unterwerfung Karthagos und der griechischen Staaten. München 51993, Bd. 2, 396-401.

12 Schmitt Pantel/Wagner-Hasel, La femme antique entre un "demi-esclavage" et une "fausse émancipation" (im Druck); WAGNER-HASEL, Rationalitätskritik 1992, 316 f. Weitere Beispiele bei Sмiтн, The Gender of History 1998; Rez. Rebekka Habermas. Historische Anthropologie 7/3, 1999, $491 \mathrm{f}$.

13 Vgl. den jüngsten Forschungsüberblick von ScheER, Forschungen über die Frau in der Antike 2000). 
schal den Frauen in der Antike, ${ }^{14}$ so folgten in den späten $80 \mathrm{er}$ und in den 90er Jahren zahlreiche Aufsatzsammlungen und Spezialstudien, die Einzelaspekte aufgriffen. Ein Großteil der Forschungen gilt den Repräsentationsweisen des Weiblichen in der antiken Literatur, dem männlichen Blick, ${ }^{15}$ aber auch den darin eingeschriebenen weiblichen Diskursen, der verdeckten Rede der Frauen. ${ }^{16}$ Vor allem sind es die weiblichen Kultpraktiken und Mythen, die Mädchen- und Frauenkulte sowie die Totenrituale, um deren Erhellung ein großer Teil der Neuerscheinungen bemüht ist; ${ }^{17}$ ebenso sind die Vorstellungen von Sexualität und vom weiblichen Körper Gegenstand aktueller Arbeiten. ${ }^{18}$ Das heiß umkämpfte Feld der Norm- und Traditionsbildung, der Machtausübung und Herrschaft wird erst in allerjüngster Zeit vermehrt in die Betrachtung einbezogen. ${ }^{19} \mathrm{Neu}$ ist das Interesse an den Vorstellungen von Männlichkeit, zu denen in den letzten Jahren eine Reihe von Studien vorgelegt wurden. ${ }^{20}$

Auch dieses neue Interesse an >Frauengeschichte ist eng mit sozialen Bewegungen verbunden. War es um die Jahrhundertwende die Frauenwahlrechtsbewegung, die neue Forschungen stimuliert hat, so bildete in den 70er Jahren die neue Frauenbewegung mit ihrer Forderung nach Chancengleichheit und Beseitigung struktureller Benachteiligung von Frauen in westlichen Industriegesellschaften einen wesentlichen Motor der Erforschung der Geschichte der Frauen. In den USA hat sie zu einer institutionellen Verankerung der Frauengeschichte in Form von $W_{0}$ -

14 Dazu gehört Sarah Pomeroys Studie Goddesses, Women, Whores and Wives von 1974, die 1985 ins Deutsche übersetzt wurde, sowie die beiden Bände von Wolfgang Schuller zu Frauen in der griechischen Geschichte (1985) und Frauen in der römischen Geschichte (1987).

15 Vgl. Schmitt Pantel, Geschichte der Frauen, Bd. 1: Antike 1993; Loraux, Les enfants d'Athèna 1990; Rollinger/Ulf, Geschlechterrollen und Frauenbild 1999.

16 Winkler, Der gefesselte Eros 1994; Papadopoulou-Belmehdi, Le chant de Pénélope 1994; Rousselle, Observation féminine 1980; Wagner-Hasel, Die Macht der Penelope 1997.

17 Blundell/Williamson, The Sacred and the Feminine 1998; Foxhall, Women's Ritual and Men's Work 1995, 97-110; Lyons, Gender and Immortality 1997; SoJc, Die (Un)sichtbaren 1999.

18 Dean-Jones, Women's Bodies 1994; King, Hippocrates' Woman 1998; Montserrat, Changing Bodies, Changing Meanings 1998; PORTER , Constructions of the Classical Body 1999; Waldner, Sexualität zwischen Reinheit und Befleckung 1998, 89-102.

19 Etwa Bartman, Livia 1998; Corbier, Male power 1995; Eisen, Amtsträgerinnen im frühen Christentum 1996; Garlick/Dixon/Allen, Stereotypes of Women in Power 1992; Kunst, Zur sozialen Funktion der Domus 1998; Osborne, Sculpted men 1998; Savunen, Women and Elections 1995; Setälä/SavunEN, Female Networks 1999; Späth, Entmachtung als Entmännlichung 1998; VAN BREMEN, Limits of Participation 1996; WOOD, Imperial Women 1999; Wieber, Zwischen Polemik und Panegyrik 1998.

20 Foucault, L'usage des plaisirs 1984; Foucault, Le souci de soi 1984; Foxhall, Foreign Power 1999; Foxhall/Salmon, Thinking Men 1998; Foxhall/Salmon, When Men were Man 1998; Meyer-Zwiffelhoffer, Im Zeichen des Phallus 1995; Rouselle, Porneia 1983; SPÄTH, Männlichkeit und Weiblichkeit 1994; SPÄTH, Männerfreundschaften - politische Freundschaften 1997; Stadter, Male Sexual Behaviour 1995; StÄHLI, Verweigerung der Lüste 1999; Veyne, La famille et l'amour 1978. 
men's Studies und zu einer Integration von akademisch ausgebildeten Frauen in die universitäre Lehre geführt, die bislang ohne Beispiel ist. Dieser Institutionalisierungsprozess der Women's Studies hat seinerseits den Bedarf an Überblicksstudien geweckt, die in Bachelor-Studiengängen als Lehrbuch einsetzbar sind. ${ }^{21}$ Ebenso ist die Entwicklung des Untersuchungsansatzes der Geschlechtergeschichte seit den 80er Jahren nicht nur auf theoretische Reflexion und die Debatten innerhalb der Frauenforschung zurückzuführen; sie erhielt auch einen starken Impuls, vor allem in der englischsprachigen Forschung, durch die Schwulen- und Lesbenbewegung und durch deren Infragestellung der vermeintlich biologisch determinierten $\mathrm{Ge}$ schlechterrollen. ${ }^{22}$

Die enge Verknüpfung von sozialer Bewegung und Forschung hat auch ihre Tücken. Ihr ist eine Benachteiligungsrhetorik geschuldet, die sich unabhängig von der jeweils verfolgten Intention in vielen Darstellungen zum Frauenleben in der Antike findet. Indem als Messlatte individuelle Autonomie, Berufstätigkeit, Bewegungsfreiheit, sexuelle Selbstbestimmung, rechtliche Gleichstellung, also all jene modernen Errungenschaften, die in der Gegenwart von Frauen erkämpft werden mussten, an das Leben von Frauen in der Antike angelegt wurde, geriet Frauengeschichte allzu leicht zur Defizitgeschichte, zur Vorgeschichte der Emanzipation bzw. des noch nicht Erreichten an sexueller Freiheit und politischer Mitbestimmung. Strukturelle Unterschiede zwischen Antike und Moderne, auf die in der struktur- und sozialgeschichtlichen Forschung aufmerksam gemacht worden war, blieben vielfach ausgeblendet. Deutlich wird dies z.B. in der Zuordnung der Frauen zum privaten häuslichen Bereich, die ein zähes Erbe der Geschichtsbetrachtung des 19. Jh. ist und erst über den Umweg der sozial-anthropologischen Forschung in der althistorischen Frauenforschung erschüttert wurde. ${ }^{23}$

Diese `Erblast « ruht auch auf dem dichotomischen Schema von Wildheit und Zivilisation, das in der Ethnologie des 19. Jh. entwickelt worden ist und über die strukturalistische Mythendeutung Eingang in die altertumswissenschaftliche Genderforschung fand. In der jüngsten Adaption dieses Gegensatzes sind Wildheit und Zivilisation, mit denen man einst kulturelle Entwicklungsstufen zu fassen suchte, im weiblichen Geschlechtscharakter vereint. Ein Beispiel für diese Sicht lieferte die Ausstellung "Pandora. Frauen im klassischen Griechenland", die 1995 in Baltimore und

21 Aus diesem Interesse ist beispielsweise das von einer Gruppe von AutorInnen (Elaine Fantham, Helene Peet Foley, Natalie Boymel Kampen, Sarah B. Pomeroy, H. Alan Shapiro) verfasste Werk Women in the Classical World: Image and Text (1994) entstanden. Es bietet einen chronologischen Überblick über Frauenleben in der antiken Welt, in den Quellenmaterial, Texte und Bilder integriert sind.

22 Vgl. etwa DuBors, Sappho is Burning 1996; Halperin, One Hundred Years of Homosexuality 1990; Halperin/WinkLer/Zeituin, Before Sexuality 1990; Konstan/Nussbaum, Sexuality in Greek and Roman Society 1989; WinkLer, Der gefesselte Eros 1994. Die Debatte über eine Pluralität von Geschlechteridentitäten und um eine entsprechend radikale Absage an den Begriff einer Geschlechterdichotomie lösten vor allem die Werke von Judith Butler (Gender Trouble 1990; Bodies That Matter 1993) aus.

23 Zur jüngsten Debatte in den Altertumswissenschaften vgl. den Tagungsband Public et Privé in: Ktema 23, 1998; SCHEER, Forschungen über die Frau 2000, $167 \mathrm{f}$. 
1996 im Basler Antikenmuseum zu sehen war. Das Ausstellungskonzept beinhaltete eine strikte Zweiteilung: Im Erdgeschoss waren im hellen Licht Exponate ausgestellt, die der "gezähmten" Frau gewidmet waren - Vasenbilder zeigten vielfach Szenen aus dem Hochzeitsritual: das Schmücken der Braut, den Hochzeitszug, den Empfang von Geschenken im Kreis der Freundinnen. Diesem Bild von der in der Ehe gezähmten Frau wurde das Bild der ungezähmten, wilden Frau gegenübergestellt, der kriegerischen Amazonen, der wilden Mänaden, die Tiere zerreißen und roh verzehren, der zauberkundigen Frauen wie Medea oder Kirke. Exponate, die derartige mythologische Figuren darstellen, waren im Untergeschoss ausgestellt und in ein geheimnisvolles Dunkel getaucht. ${ }^{24}$ Nach dem Konzept der Ausstellungsmacherinnen - und in diesem Deutungsversuch liegt das einzig Neue an der traditionellen Gegenüberstellung "weiblicher Natur" und »männlicher Kultur» drückten diese Figuren die Angst der griechischen Männer vor dem Wilden, dem Ungezähmten und der sexuellen Verführungsmacht der Frau aus. Das Konzept selbst fußt auf einer Tradition der Konzeptualisierung von Geschlechterrollen, wie sie etwa in Johann Jakob Bachofens Werk Das Mutterrecht zu finden ist, wo diese Gegensätze von sexueller Verführungsmacht und gezähmter Sexualität noch in ein historisches Nacheinander gestellt und als Abfolge gynaikokratischer Stufen vom Amazonen- und Hetärentum zum demetrischen Muttertum dargeboten sind. ${ }^{25}$

Diese unkritische Haltung gegenüber der Konstruktion einer a-historischen, universalen ,Weiblichkeit $\iota$, auf deren Grundlage Frauen der Antike betrachtet werden, ist umso verwunderlicher, als die sEntdeckung der 'Geschichtlichkeit der Frauen zu den wichtigsten Einsichten der jüngsten Frauengeschichte zählt. Sie ging einher mit einer konsequenten Historisierung der Kategorien Öffentlichkeit und Privatheit sowie Natur und Kultur und ist Teil eines Perspektivenwechsels von der Politik- zur Mentalitäten-, Alltags- und Kulturgeschichte. Ausgehend von dem durch französische Historiker seit den 30er Jahren des 20. Jh. aufgestellten Postulat einer "longue durée», mit dem die "Mentalitäten" zu Objekten der historischen Forschung wurden, zielen die neuen Forschungen auf eine historische Anthropologie, in der auch das vermeintlich Beständige in seiner Wandelbarkeit untersucht

24 "Im viertem Abschnitt endlich geht es um die bis zur Katastrophe gesteigerten mythischen Ausformungen jener anhaltenden Angst, dass der Zähmungsprozess der Frauen scheitern könnte, dass die den Frauen angeborenen Impulse sich schließlich wieder durchsetzen würden. Bestimmte, den Frauen zugeschriebene Verhaltensweisen haben tiefeingewurzelte Ängste hervorgerufen, die bis in die Neuzeit weiterleben: unkontrollierte Raserei, die Frauen dazu antrieb, Männer zu morden (Orpheus und die Thrakerinnen, Mänaden); völlige Ablehnung einer männlich-zentrierten sozialen Struktur (Amazonen); die Fähigkeit, Männer ihrer Stärke und ihrer Männlichkeit zu berauben (Eros und Kephalos); und die unfassbare und unwiderstehliche Gewalt des Blickes (Gorgonen), der Stimme (Sirenen), sowie der Macht über giftige Substanzen (Medea und Kirke)." ReEDER, Pandora 1995/1996, I8.

25 Wagner-Hasel, Rationalitätskritik 1992; Hartmut Zinser, Mythos des Mutterrechts 1981. 
wird. ${ }^{26}$ Für die Mediävistin und Renaissanceforscherin Gianna Pomata, die Anfang der 80er Jahre in ihrer Studie Die Geschichte der Frauen zwischen Anthropologie und Biologie für eine konsequent historische Sicht plädierte, ist es die begriffliche Zuordnung der Frauen zu den vermeintlich anthropologischen Konstanten menschlichen Daseins wie Geburt, Heirat, Tod, die ihnen eine marginale Rolle in der Geschichtswissenschaft zugewiesen hatte. Für sie ist diese Verortung ein Erbe des Gegensatzes zwischen Anthropologie und Geschichte, der mit der Entstehung der "wissenschaftlichen Geschichtsforschung" im 19. Jh. aufkam, und impliziert eine Mystifizierung eines vermeintlich Archaischen in einer sich rapide verändernden Umwelt. ${ }^{27}$ Damit erklärt sie die Verortung der Frauen in der Nähe von Natur, während der Mann aufgrund seiner Verknüpfung mit den großen Neuerungen des Jahrhunderts wie Industrialisierung oder Nationalstaatsbildung dem Bereich der Kultur zugeordnet worden sei. Während die Debatten um die Universalien wie Kultur und Natur, Öffentlichkeit und Privatheit in den 70er und 80er Jahren vor allem in Anthropologie und Geschichtswissenschaft geführt wurden und die Kategorien eine $>$ Historisierung erfuhren, ${ }^{28}$ kommt in den Altertumswissenschaften

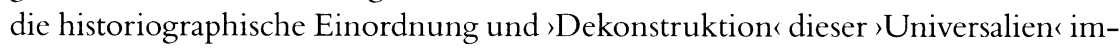
mer noch zu kurz, auch wenn in den letzten Jahren das Interesse an der Historiographie deutlich größer geworden ist. ${ }^{29}$

Gianna Pomata gehört mit ihrer kritischen Sicht auf die vermeintliche Naturhaftigkeit des Weiblichen zu den frühen Vertreterinnen einer Geschichtsbetrachtung, die nicht nur auf der Geschichtlichkeit der Erfahrung von Frauen insistierte, sondern sich auch auf die Seite derjenigen schlug, die bereits in den 70er Jahren 'Geschlechtsidentität als Kategorie des historischen Forschens wie Klasse, Stand oder Schicht gefordert hatten. Diese Konzeptualisierung von Geschlecht als historische Kategorie gehört zu den innovativsten Ansätzen der letzen drei Jahrzehnte.

26 Damit löst sich der diffuse Begriff der "Mentalitäten« allmählich auf. Vgl. die radikale Kritik des Mentalitätenkonzepts aus der Sicht des Hellenisten Geoffrey E. R. Lloyd, Demystifying Mentalities 1990.

27 Pomata, Frauen zwischen Anthropologie und Biologie 1983, 117. Als Beispiel für diese Zuordnung der Frauen zu einem Bereich des "Nicht-Historischen innerhalb der historischen Welt« zitiert sie aus de Martinos Beschreibung ritueller Formen der mediterranen Totenklage Morte e pianto rituale aus dem Jahre 1958. In ihr fungierten die Frauen als Vehikel des Überdauerns des Archaischen, als unhistorische Wesen, "die snur die Irrationalität der Naturkräfte und die erdrückende Unterdrückung der gesellschaftlichen Kräfte erfahren haben «" und daher als einzig mögliche Reaktion nicht die bewusste, historische Handlung kennen, sondern nur die archaische Technik des Klageritus.

28 Zur anthropologischen Diskussion vgl. MacCormack/Strathern, Nature 1990;

Rosaldo, Use and Abuse of Anthropology 1980; Rosaldo/Lamphere, Women, Culture 1974; zur Diskussion in den Geschichtswissenschaften vgl. u.a. Medick/TrePP, Geschlechtergeschichte und allgemeine Geschichte 1998; speziell zur Altertumswissenschaft: Wagner-Hasel, Perspektive ‘Geschlecht‘ I988; WInkLer, Der gefesselte Eros 1994; WaLDNER, Geburt und Hochzeit des Kriegers 2000).

29 Allgemein: Bцок, Sexual Asymmetry. A Historiographical Essay 1987; Wagner-Hasel, Das Private wird politisch 1988; zu Griechenland: KATZ, Ideology and the Status of Women 1995.; zu Rom: SpäTH, Frauenmacht 1994. 
Sie reflektiert zum einen die kulturelle Determiniertheit von Geschlechterrollen und diente zunächst der Abgrenzung vom biologischen Geschlecht. Die historisch-gesellschaftlichen Erscheinungsformen von Weiblichkeit und Männlichkeit wurden in der englischsprachigen Forschung als gender bezeichnet und vom vermeintlich a-historischen, biologisch bestimmten sex` abgegrenzt. In der Folge historisierte die Frauenforschung aber ebenso das biologische Geschlecht, indem die theoretischen Arbeiten postulierten, auch was eine bestimmte Gesellschaft einer bestimmten Epoche der `Natur ' zuordne und was sie darunter verstehe, sei kulturell determiniert. ${ }^{30} \mathrm{Nach}$ Auffassung der amerikanischen Historikerin Joan W. Scott, die die Debatte maßgeblich geprägt hat, "[ist] das so genannte biologische Geschlecht $[\ldots]$ genauso eine historisch und kulturell variable Form von Wissen wie die Vorstellungen über Geschlechterrollen" und wird "die Unterscheidung zwischen Frauen und Männern in Begriffen der körperlichen Differenz überhaupt erst geschaffen ${ }^{31}$ Geschlecht bezeichnet demnach nicht Fakten, sondern Bedeutungen: ${ }^{32}$ Nach Scott kann der Begriff bestimmt werden als »die vielfältigen Bedeutungen, die sexueller Unterschiedlichkeit zugeordnet werden ${ }^{33}$. Sie umschreibt diese Herausbildung von Geschlecht durch die Zuordnung von Unterschieden in vier Aspekten: Geschlecht besteht aus kulturell verfügbaren Bildern, die in Begriffen (wie 'Selbstbeherrschung « $>$ Maßlosigkeit<, >Reinheit und >Beschmutzung`) und in symbolisierten Figuren (wie `Eva`, 'Maria‘, ’Herakles`, 'Salomon`) Ausdruck finden. Die Bedeutungsvielfalt dieser Symbole wird durch normative Konzepte eingeschränkt, die verschiedenen Geschlechteridentitäten (beispielsweise dem aristokratischen Mann, der Sklavin, dem Freigelassenen, der jungen, unverheirateten Frau) zugeordnet werden. Die sozialen Institutionen und Organisationsformen sind das dritte Element der Geschlechterdefinition: in Verwandtschaftssystemen etwa, im Rahmen von bestimmten Erziehungsformen, ökonomischen und politischen Strukturen bilden sich die genannten Bilder und Konzepte heraus und werden tradiert. Schließlich definiert sich Geschlecht in der sozialen Konstruktion subjektiver Identitäten: die jeweilige »individuelle Identität als Frau, als Mann (oder in weiteren Differenzierungen: als Gattin in einem aristokratischen Haus, als Sklavin etc.) ist Ergebnis von gesellschaftlichen Praktiken und wird durch diese gefestigt.

30 Zum Begriff und seiner Entwicklung vgl. Egger, Gender 2000, Abschnitt A. Zum Nachweis von Biologie als gesellschaftlich bestimmter Kategorie vgl. Воск, Geschlechtergeschichte 1988, 374 ff. Dass ")Natur` und sex Konzepte mit Geschichte sind (SCOTt, More Reflections 1999, 200), weist insbesondere Butler, Bodies 1993 nach. Aus diesem Grunde postulieren Bock, International Debate 1989, NiCHOLSON, Interpreting 1994 und Hawkesworth, Confounding Gender 1997 die Aufgabe der Begriffsunterscheidung von gender und sex.

31 Scott/Arni, Politik der Geschichte 2000, 24.

32 Die folgenden Darlegungen fassen zusammen, was in SPÄTH, Entmachtung als Entmännlichung 1998, 8 ff., ausgefuhrt wird.

33 Sсотт, Gender 1988, 25 (hier wie im folgenden bei nicht-deutschsprachigen Zitaten: unsere Übersetzung). 
Diese vier Elemente definieren nach Scott - in jeweils historisch-kulturell bestimmter Ausprägung und Kombination - Geschlecht und die geschlechterspezifische Struktur einer Gesellschaft. Vor dem Hintergrund dieser Prämisse konnte Geschlecht als ein "konstituierendes Element gesellschaftlicher Beziehungen «" ${ }^{34}$ und damit als historische Kategorie gefasst werden, die gemeinsam mit anderen Faktoren - wie Rechtsstatus, Klassen- oder Standeszugehörigkeit, ökonomischen Produktionsbedingungen - die primäre Grundstruktur der gesellschaftlichen Ordnung bestimmt. Das heißt: Geschlecht wurde zu einer Kategorie der allgemeinen historischen Forschung erhoben und nicht mehr auf ein Teilgebiet, das Leben der Frauen oder die Geschichte der Sexualität, bezogen.

Gegenüber der historischen Frauenforschung beinhaltete das Konzept der Geschlechtergeschichte eine Erweiterung insofern, als nun auch die geschlechterspezifische Bestimmung männlichen Handelns und die gesellschaftlichen Normen der Männlichkeit ins Blickfeld der Forschung gerieten. Damit wurde auch den Begriffen Frau und Mann eine a-historisch allgemeine Bedeutung abgesprochen und die Forderung aufgestellt, an jede Kultur und jede Epoche immer wieder neu die Frage zu stellen, was sie als Geschlechterunterschiede wahrnimmt und welche Bedeutungen sie diesen Unterschieden zuordnet, um auf dieser Grundlage Geschlechtsidentitäten zu definieren. ${ }^{35}$ Hinzu kommt eine dritte Dimension, die Bedeutung von `Geschlecht als Strukturkategorie. Ivan Illich hat in seinem wenig rezipierten Buch Genus von 1983 das Konzept für eine Theorie der vormodernen Gesellschaften genutzt und Material für seine These zusammengetragen, dass in vormodernen Gesellschaften `Geschlecht` eine weit größere Rolle zur sozialen Strukturierung der Gesellschaft gespielt habe als in der Moderne. ${ }^{36}$ Die in der Alten Geschichte zunehmend angewendeten anthropologischen Konzepte von der Segregation der Sphären der Geschlechter basieren nicht zuletzt auf einer solchen Vorstellung der Gliederung sozialer Räume nach geschlechterspezifischen Kriterien. ${ }^{37}$

In den Altertumswissenschaften verband sich die Aufnahme geschlechterspezifischer Ansätze mit einem Methodenwechsel und einer Veränderung der Fragestellung. War die Erforschung von Frauenleben in den 70er Jahren primär von sozial-

34 ScOTt, Useful Category 1988, 42: "gender is a constitutive element of social relationships based on perceived differences between the sexes". Zum Folgenden vgl. $43 \mathrm{ff}$.

35 Vgl. Pomata, Storia delle donne 1983 und ihr Postulat der Ersetzung monothetischer Begriffe (wie die Frau, der Grieche, die Römerin) durch polythetische, die von einer Bedeutungsvielfalt ausgehen. Gegen Forschungen nach einer vermeintlich "ursprünglichen Weiblichkeit" WAGNER-HASEL, Rationalitätskritik 1992, Anm. 12, 339, mit Literaturhinweisen; vgl. auch die Angaben in SCHEER, Forschungen 2000, $148 \mathrm{f}$.

36 ILLICH, Genus 1983. Aus historisch-anthropologischer Sicht ist in Illichs These der universale Geschlechterdualismus problematisch, den er für vorindustrielle Gesellschaften postuliert; sozialanthropologische Forschungen können demgegenüber unterschiedlichste Formen des Spiels mit Geschlechteridentitäten und damit gerade eine Vielfalt nachweisen, welche die vermeintlich fixe "Genus-Dualität» von Illich in Frage stellt. Vgl. etwa BRETON, La mascarade des sexes 1989.

37 Vgl. u.a. Wagner-Hasel, Das Private wird politisch 1988; Foxhall, Gender 1989, Sourvinou-INwood, Männlich und weiblich 1996. 
geschichtlichen Methoden bestimmt und versuchte man, den literarischen Überlieferungen Informationen über das reale Leben der Frauen zu entnehmen, so wurde unter dem Einfluss der Geschlechter-Debatte eben dieses Vorgehen zum Problem. Im Unterschied dazu bevorzugen Studien über die gesellschaftliche Definition der Geschlechter und die Vorstellungen von Weiblichkeit und Männlichkeit diskursanalytische Verfahrensweisen, die sich auf die vor allem von Michel Foucault entwickelte Diskurstheorie stützen. ${ }^{38}$ Diskurse betreffen nach Foucault keineswegs nur sprachliche Äußerungen, sondern umfassen die Bedingungen sozialer Praktiken. Im Rahmen dieser Diskurstheorie sind Geschlechterdiskurse als ein Ensemble von Regeln zu verstehen, die darüber bestimmen, auf welche Weise Geschlechterdifferenzen und -definitionen in einer gegebenen historischen Situation erkennbar, denkbar, sagbar und handlungsorientierend sind. Diese Regeln stehen nicht ein für allemal fest, sondern bilden sich in der gesellschaftlichen Praxis: jede Umsetzung stellt sie in Frage und verändert sie. Indem die diskursiven Regeln das Ereignis bestimmen und zugleich vom Ereignis bestimmt werden, zeichnet sich die Diskurstheorie, worauf sich auch Scotts Bestimmung von Geschlecht bezieht, als eine post-strukturalistische Theorie aus. ${ }^{39}$

Post-strukturalistische Theorien sind vor allem in den Literaturwissenschaften ausgebildet und zum Teil in den Altertumswissenschaften rezipiert worden. Die englische Althistorikerin Averil Cameron führt für die Affinität althistorischer Geschlechterforschung zu diesen Ansätzen zwei Gründe an: Bei den Texten, mit denen die Altertumswissenschaften arbeiten, gehe es um einen 'klassischen Kanon', dessen Nutzung durch eine weit zurückreichende philologische und althistorische Tradition gleichsam vorgegeben sei. Die post-strukturalistischen Ansätze bezeichnet sie als eine "subversive Form moderner Literaturtheorie«, womit sich die von dieser Tradition auferlegte 'Ehrfurcht durchbrechen und der klassische Kanon 'gegen den Strich lesen lasse. Andererseits sieht Cameron die Anwendung poststrukturalistischer Methoden in der Quellenlage begründet: die Frauen- und Geschlechterforschung im Bereich der Antike könne sich nicht auf die traditionelle positivistische Herangehensweise beschränken; Frauen und Geschlechterdefinition seien nicht Themen der griechisch-römischen Literatur, die zudem nahezu ausschließlich durch Männer geschrieben und durch Männer überliefert sei; mit einer rein empirischen Lektüre antiker Texte ließen sich auf geschlechterspezifische Fragen keine Antworten finden..$^{40}$ Im Gegensatz dazu geht eine diskursanalytische

38 Foucault, Archéologie 1969, 55-93. Dazu: SpÄтн, Geschlechter 1994, 285-289.

39 Vergleichbar damit ist der Begriff der kulturellen Schemata, wie ihn Sahuins, Islands 1985 festlegt: jede Umsetzung von Elementen dieses kulturellen Schemas in soziale Praktiken verändere das Schema und dessen Konzepte (vgl. zu diesem Aspekt den Kommentar von LenCLud, Sahlins 1991). Post-strukturalistische Theorien gehen von strukturalistischer Grundlage aus, überwinden aber die schematische Dichotomie des klassischen Strukturalismus (Struktur versus Praxis nach dem Modell der Unterscheidung von langue und parole durch Saussure), indem sie die notwendig wechselseitige Beziehung von Struktur und Praktiken thematisieren.

40 Cameron, Women in Antiquity 1989, 10. 
Lektüre davon aus, dass jeder Schreibende die Sprache und die Konzepte seiner historisch-kulturellen Situation verwendet: Wenn ein Homer oder Aristophanes, ein Cicero oder Tacitus von Männern und Frauen schrieben, so beschrieben sie nicht deren Weiblichkeit und Männlichkeit, aber sie stellten sie dar mit den Vorstellungen von Männlichkeit und Weiblichkeit, die ihnen zur Verfügung standen. ${ }^{41}$ Aufgrund dieser theoretischen Prämisse postuliert eine solche Lektüre, jeder Text lasse sich auf seine unterschiedlichen Bedeutungsebenen ${ }^{42}$ hin untersuchen unter der Voraussetzung, dass seine erste, poetisch-erzählerische Ebene analysiert werde im Sinne einer Auflösung seines Erzählstrangs, um die oben skizzierten Elemente einer Geschlechterdefinition, die Begriffe und Bilder, die normativen Konzepte, institutionellen Formen und Geschlechteridentitäten zu isolieren. Eine solche 'Zerstörung` oder `Destruktion` der vordergründigen Bedeutungsebene des Textes ist Grundlage, um seine geschlechterspezifische Bedeutungsebene zu ’konstruieren . In diesem sehr einfachen Sinn kann diese Lektüremethode als `Dekonstruktion verstanden werden.

Was eine diskursanalytische oder in diesem Sinn >dekonstruktivistische< Lektüremethode aus den Texten der griechisch-römischen Antike gewinnen kann, ist der Geschlechterdiskurs in seinem jeweiligen historischen Kontext. Die rgelebte Wirklichkeit einzelner Männer und Frauen bleibt diesen Lektüremethoden verschlossen; die Untersuchung des Geschlechterdiskurses ermittelt den historischen Rahmen, worin Männer und Frauen dachten, redeten und handelten, sie erfasst die Bedingungen gesellschaftlicher Praktiken, nicht aber diese Praktiken in ihrer historischen Ereignishaftigkeit. ${ }^{43}$

Genau hier setzt die Kritik an von Forscherinnen, die als Aufgabe der Frauengeschichte die Rekonstruktion der Realität vergangenen Frauenlebens postulieren. Die heute keineswegs abgeschlossene Debatte dreht sich allerdings nicht nur um den Nutzen bestimmter Lektüremethoden, sondern stellt die Konzepte von Frauengeschichte der Geschlechtergeschichte gegenüber. Exemplarisch kann eine Auseinandersetzung diese Problematik umreißen, die vor einigen Jahren die amerikanische Geschlechterforschung bewegte: Im Jahre 1985 schlug der Women's Classical Caucus (WCC) das Thema "Reappropriating Male Texts: The Case of Ovid" ("Männliche Texte zurückgewinnen: der Fall Ovid«) für seinen Workshop an der Jahresversammlung der ehrwürdigen American Philological Association vor. ${ }^{44}$ Die Al-

41 Ausführlicher dazu SpäTh, Texte 1994, 11-33.

42 Zur Unterscheidung der Bedeutungsebenen der Texte vgl. Kristeva, $\Sigma \eta \mu \varepsilon \imath \omega \tau \imath \kappa \eta 1969$, $11,52,85,120,218$.

43 Geschlechterdiskurs kann deshalb nicht auf "Geistesgeschichte " reduziert werden, und aus einer geschlechtertheoretischen Sicht »entstammen« literarische Quellen aus diesem Grunde nicht nur, wie PAтZeK, Quellen 2000, 10, schreibt, »dem Denken«, sondern genauso den Bedingungen der "Alltagspraxis".

44 Das Organisationskommitee der APA ("five senior classicists who are appointed by the Directors of the association from within their own ranks") lehnte den Workshop ohne weitere Begründung als »simply not acceptable«ab. Die Organisatorinnen des Workshops unter Leitung von Mary-Kay Gamel führten ihn gleichwohl durch - mit dem großen 
thistorikerin Phyllis Culham reagierte darauf mit einem polemischen Beitrag: Das zentrale Anliegen moderner feministischer Wissenschaft sei die Entdeckung der "gelebten Wirklichkeit von Frauen«. Eine Gruppierung mit feministischer Ausrichtung wie der WCC dürfe es nicht zulassen, mit der Ausrichtung auf literarische Texte die materielle Kultur und damit die übergroße Mehrheit der arbeitenden Frauen auszugrenzen. Das feministische dekonstruktivistische Interesse an "von Männern verfassten Texten« verstärke nur die schöngeistig-klassizistische Überschätzung des "ahistorisch bleibenden" Wertes der kanonischen literarischen Texte. Culhams Folgerung: Feministische Wissenschaftlerinnen könnten sich nicht erlauben, in einer Zeit politischer Reaktion - die USA hatten zu jener Zeit Ronald Reagan als Präsidenten - ihre beschränkten Ressourcen auf eine Erörterung von Stellung und Wert männlicher Autoren des klassischen Kanons zu verschwenden. ${ }^{45}$ Mary-Kay Gamel weist in ihrer Antwort darauf hin, Culham weise keinen Weg, wie von materiellen Überresten auf die "gelebte Realität" von Frauen geschlossen werden könne: »jede Form von Dokumenten, ob literarisch oder nicht-literarisch, sind Texte und verlangen als solche komplexe Techniken der Lektüre«. Das Forschungsergebnis "gelebte Wirklichkeit« könne deshalb nur eine durch Texte konstruierte Wirklichkeit sein. ${ }^{46}$

Doch mit ihren Forderungen steht die amerikanische Historikerin Culham keineswegs allein: Eine der Pionierinnen antiker Frauengeschichte, Sarah B. Pomeroy, meinte vor einiger Zeit eine historisch-anthropologische Untersuchung wie jene von Giulia Sissa zum Begriff der Jungfräulichkeit im klassischen Griechenland ${ }^{47}$ mit einem Argument aburteilen zu können, das sie bezüglich einer Vielzahl von Studien zur antiken Frauengeschichte verallgemeinerte: "Manche Möchtegern-FrauenhistorikerInnen, die Frauenleben aufgrund der Untersuchung von Männerideen über Frauen erforschen wollten, sind eher in literarischer als in historischer Methodologie ausgebildet. Sie nennen sich `SozialhistorikerInnen` in der Meinung, Sozialgeschichte sei eine weniger rigorose Disziplin als politische oder Wirtschaftsgeschichte. ${ }^{48}$ Und Mary Lefkowitz warnte vor dem exklusiven Gebrauch literarischer Dokumente, speziell von Tragödien und Gerichtsrhetorik, zur Rekonstruktion eines Bildes der Alten Welt. ${ }^{49}$ Sie wirft feministischer Theorie vor, zwar "Frauen ansprechende, aber absurde und in die Irre führende Interpretationen be-

Erfolg zahlreicher Beteiligung und engagierter Diskussionen. Auf diesen institutionellen

Konflikt und seine Konsequenzen soll hier nicht weiter eingegangen werden, vgl.

GaMEL, Reading Reality 1990.

45 Culham, Decentering 1990, $161 \mathrm{f}$.

46 Gamel, Reading Reality 1990, 171. Zur Debatte vgl. neben diesem Beitrag auch in der gleichen Nummer der Zeitschrift Helios Cahoon, Poetry, Criticism, Feminism 1990; French, What Is Central 1990; Hallett, Contextualizing 1990; Keuls, Feminist View 1990; Manus, Athenian Bride 1990; Richlin, Palladion 1990.

47 Sissa, Corps virginal 1987.

48 Pomeroy, Study of Women 1991, $265 \mathrm{f}$.

49 LefKowitz, Myth 1986, 28; vgl. dazu die differenzierte Kritik in der Rezension von Jane Cahill, in: Phoenix 43, 1989, 165-169, speziell 166. 
kannter Texte hervorgebracht" zu haben, während "wichtige neue Erkenntnisse über die Stellung der Frauen der Antike hervorgebracht wurden mit traditionellen Mitteln der Forschung, und sehr oft durch Männer" - und dieser Vorwurf richtet sich spezifisch gegen postmoderne Literaturtheorien, welche die klassischen Texte gegen den Strich lesen. $^{50}$

In weniger polemischem Ton, aber nicht minder deutlich in der Sache wirft Claude Mossé dem ersten Band der Geschichte der Frauen vor: "wenn man zu viel vom Weiblichen redet, läuft man Gefahr, die Frauen in ihrer konkreten Realität aus den Augen zu verlieren und die Tatsache zu übersehen, dass unter ihnen, genauso wie unter den Männern, Unterschiede des Status, des Vermögens, der Tätigkeiten bestanden, welche nicht völlig durch ihre gemeinsame Lebensbedingung, Frau zu sein, verwischt wurden. ${ }^{51}$ Und Gianna Pomata hält in ihrer Kritik an den Beiträgen zum Mittelalter und zur Neuzeit der Geschichte der Frauen fest, Geschlechtergeschichte sei nützlich als eine Geschichte der gesellschaftlichen Konstruktion der Kategorien des Männlichen und des Weiblichen, sie dürfe aber "nicht verwechselt werden mit der Frauengeschichte, und keinesfalls die Notwendigkeit einer Sozialgeschichte der Frauen überschatten. ${ }^{52}$

Die Auseinandersetzungen um Frauen- und Geschlechtergeschichte greifen grundlegende geschichtstheoretische Fragen auf: Lin Foxhall warnte in ihrer Kritik an der Diskurstheorie Foucaults davor, die literarischen Produkte eines männlichen Selbst allzu wörtlich und den Ausschnitt, den sie von der antiken Welt liefern, für das Ganze zu nehmen. ${ }^{53}$ Foxhalls Anliegen ist es, die nicht-hegemonialen Diskurse einzubeziehen und sowohl die Bedeutung von Handlungen als auch von Vorstellungen in ihrer Widersprüchlichkeit zu analysieren. So stellten Verwandtschaftsideologien in der griechischen Antike zwar das männliche Ego in den Mittelpunkt; nichtsdestoweniger sei aber erkennbar, dass die Frauen für das Funktionieren von verwandtschaftlichen Beziehungen über ihre Zugehörigkeit zu zwei verschiedenen Haushalten von maßgeblicher Bedeutung seien. ${ }^{54}$ In ähnlicher Weise fordert die Klassische Philologin Katharina Waldner, die symbolische Ordnung in einen Dialog zu bringen mit dem Wissen über Institutionen wie Heiratsregeln und Arbeitsteilung, über religiöse Bräuche und deren Funktion bei der Organisation des Zusammenlebens der Geschlechter. ${ }^{55}$ Neuzeithistorikerinnen wie Kathleen Canning, Mitherausgeberin der Zeitschrift Gender \& History, und Rebekka Habermas machen darauf aufmerksam, dass man mit diskurstheoretischen

50 Lefkowitz, Feminism 1983, zitiert in Richlin, Palladion 1990, 178 und 183, als Beispiel für "women in classics [who] have simply accepted the male identification natural to such an old-boy field".

51 Mossé, L'antiquité: Lecture critique 1993, 21.

52 Pomata, Histoire des femmes, histoire du genre 1993, $29 \mathrm{f}$.

53 Foxhall, Pandora unbound 1994; sie bezeichnet ihr eigenes Vorgehen als Ethnographie, "that is, a consideration of the synchronic, simultaneous, changing contexts in which conflicting (often incompatible) discourses operate" (134).

54 FoXhall, Pandora unbound 1994, 135 u. 140.

55 WaldNER, Gender Studies in den klassischen Altertumswissenschaften 1999. 
Genderkonzepten zwar historischen Wandel beschreiben, ihn aber nicht erklären könne. ${ }^{56}$ Sie sehen in der Beschränkung auf Dekonstruktion und im Verzicht auf Deutung und auf die sgroße Erzählung eines der entscheidenden Defizite der gegenwärtigen Frauen- und Geschlechtergeschichte. Canning forderte unlängst auf einer Tagung ${ }^{57}$ eine Rückkehr zu alten Kategorien wie Klasse oder Bürger und damit zur Gesellschaftsgeschichte, die aber »engendered«, unter geschlechterspezifischer Perspektive, zu fassen sei.

Was sich in diesen Auseinandersetzungen und Divergenzen zeigt, sind die unterschiedlichen Möglichkeiten, Fragen über Frauenleben und Geschlechterdefinitionen in der Antike zu stellen. Im vorliegenden Band ist keine der hier angesprochenen Ausrichtungen exklusiv vertreten. Er präsentiert vielmehr ein breites Spektrum von Fragestellungen und methodischen Zugriffen. Diskurstheoretische Ansätze stehen neben ereignisgeschichtlich ausgerichteten Beiträgen, sozialgeschichtliche Strukturanalysen neben literaturgeschichtlich-philologischen Untersuchungen einzelner Werke. Der Band ist als Arbeitsbuch konzipiert und enthält sowohl literarische als auch Bildquellen. Die einzelnen Beiträge greifen eine Problematik auf und verstehen sich als Einführung - und laden zugleich ein zu einer eingehenderen Auseinandersetzung mit den Bild- und Textquellen, wozu auch die Literaturangaben jedes Beitrags eine Hilfestellung sind. Gemeinsam ist den Beiträgen ein quellenkritischer Zugriff, über den die Interessegerichtetheit und der zeitlicher Kontext der Überlieferungen deutlich wird, auf denen unser Wissen über Frauenwelten in der Antike und die Ordnung der Geschlechter beruht. Die Darstellung ist systematisch und folgt einer chronologischen Ordnung nur insofern, als in jedem Kapitel versucht wird, den Bogen von der mesopotamischen und griechischen Frühzeit zur römischen Kaiserzeit und zum Christentum zu schlagen, wobei Auslassungen nicht ausblieben. Anliegen war es, sowohl Unterschiede in der historischen Entwicklung als auch im Vergleich zwischen vorderorientalischen, griechischen, römischen und spätantik-christlichen Kulturen deutlich werden zu lassen. Der Band zielt darauf ab, eine - wenn auch nicht homogene - Sicht von antiken Gesellschaften aus geschlechterspezifischen Perspektiven zu vermitteln.

Der Band gliedert sich in die Kapitel (I) Heirat und Zugehörigkeit, (II) Weibliche Kultpraxis, (III) Wissen und Tradition, (IV) Macht und Politik, (V) Arbeitswelt und weibliche Geselligkeit, (VI) Erotik und Sexualität. Es handelt sich um eine offene Struktur, die vom Bindungsverhältnis von Frau und Mann und vom Hauswesen ausgeht. Dieses Thema greift das erste Kapitel auf. Brigitte Groneberg stellt frühe Gesetzestexte aus Mesopotamien vor, die Aspekte des Güteraustauschs zwischen den Geschlechtern bei Heirat und Scheidung regeln. Das Verhältnis zwischen dem Bindungsverhältnis der Politen, der Polis, und dem Bindungsverhältnis

56 Vgl. Canning, History after the Linguistic turn 1994, wo sie sich mit den Postulaten von Joan Scott auseinandersetzt.

57 "Neue Horizonte? Stand und Perspektiven der Geschlechtergeschichte", Tagung an der Universität Basel vom 17. Juni 2000, organisiert von Susanna Burghartz und Claudia Opitz. 
des Paares, der Ehe, steht im Mittelpunkt des Beitrages von Elke Hartmann über die Heirat in Griechenland. Christiane Kunst untersucht die Heiratsmotive und die Bedeutung von Heirat und Scheidungen in der römischen Aristokratie. Die drei Beiträge ordnen die Bindungsverhältnisse in ihren jeweiligen gesellschaftlichen Kontext ein und zeigen die Vielfalt der historischen Realitäten, die allzu vereinfachend unter den Begriff der "Ehe« eingeordnet werden.

Die Reproduktion der gesellschaftlichen Strukturen in den rituellen Praktiken und im Kult ist das Thema des zweiten Kapitels. Die Beiträge konzentrieren sich auf kollektive Kulthandlungen von Frauen verschiedener Altersstufen, auf die rituellen Handlungen von Mädchen im Rahmen von Poliskulten, auf die Kultpraktiken der erwachsenen Frauen im Rahmen des Totenkults, auf die kultischen Verrichtungen der unverheirateten Vestalinnen in Rom und auf die Riten der Matronen bei den römischen Frauenfesten. Auch wenn die Fokussierung auf weibliche Rituale erfolgt, werden diese immer auch auf ihre Bedeutungen für die Ordnung der Geschlechter und für die gesamte Gesellschaft hin untersucht. Katharina Waldner behandelt die Rituale der BBärinnen ` im Artemisheiligtum von Brauron, die sie als Teil der Polisreligion fasst, und thematisiert u. a. deren Bedeutung für die räumliche Integration der Polis. Auf die zentrale Bedeutung der weiblichen Totenklage für den Nachruhm der Toten geht der Beitrag von Beate Wagner-Hasel ein; den gleichen Handlungsbereich greift Francesca Prescendi für die römische Kultur in ihrer Untersuchung der geschlechterspezifisch unterschiedlichen Praktiken im Totenritual auf. Hildegard Cancik-Lindemaier stellt die Frage nach der Einzigartigkeit der römischen Vesta-Priesterinnen und nach ihrer Bedeutung für den Staatskult. Um eheliche und verwandtschaftschaftliche Bindung geht es in den von Francesca Prescendi untersuchten Festen Matronalia und Matralia, die die römischen verheirateten Frauen unter sich feierten.

Das dritte Kapitel vertieft die Frage nach der Rolle der Frauen bei der Tradierung von Wissen über das Funktionieren von Gesellschaft und öffnet sie zugleich in Richtung eines breiter verstandenen Bildungsbegriffs. Christine Schnurr-Redford führt am Beispiel der delphischen Pythia vor, wie die ,Dienerinnen des Apollon Kompetenzen erwarben und ganz selbstverständlich aufgrund ihres Wissens im Mittelpunkt der Orakelbefragung standen. $\mathrm{Zu}$ den Elegien der römischen Dichterin Sulpicia legt Christine Rohweder eine Untersuchung der Bedingungen weiblichen Schreibens und von dessen Rezeption in der Klassischen Philologie vor. Sie vergleicht Sulpicias Texte auch mit den Gattungsregeln, die uns aufgrund der männlichen Dichter von Liebeselegien erschließbar sind. Die Überlieferungsbedingungen der Texte von Philosophinnen und ihre philosophischen Fragestellungen diskutiert Henriette Harich-Schwarzbauer ausgehend von den Pythagoreerinnen in hellenistischer Zeit und bis zu Hypatia, die in der zweiten Hälfte des 4.Jh. in Alexandria lebte und lehrte. In die Welt der frühchristlichen Gemeinden Nordafrikas fuihrt der Beitrag von Peter Habermehl, der das einzigartige Dokument des Tagebuchs und der Visionen von Perpetua vorstellt, die zu Beginn des 3. Jh. im Amphitheater Karthagos starb. Ruth Harder beschreibt die Situation von Anna Komnene, Tochter des byzantinischen Kaisers, die in der ersten Hälfte des 12. Jh. ein Geschichtswerk schrieb und zugleich als Mäzenin einen Kreis von Gebildeten 
um sich sammelte. Das breite chronologische Spektrum der Beiträge weist auf eine konstante Teilhabe von Frauen an der Konstruktion von Wissen und Bildung hin. Und gleichzeitig zeigen sie auch die vielfältigen Differenzen der Bedingungen, vom kultischen Kontext der Pythia bis zum ıliterarischen Zirkel um Anna Komnene, unter denen Frauen als Subjekte der Wissensproduktion agierten.

Die Untersuchung der Bedeutung von Frauen als Trägerinnen von Wissen und Vermittlerinnen von Tradition führt zur Frage des vierten Kapitels nach dem Platz von Frauen in den Strukturen der gesellschaftlichen Macht. Beate Wagner-Hasel argumentiert, dass antike Vorstellungen vom Machtverhältnis zwischen den Geschlechtern nicht mit dem modernen Patriarchatsbegriff erfasst werden können, und vielmehr eine geschlechterspezifische Macht in getrennten und komplementären Räumen in den philosophischen und literarischen Texten des griechischen Altertums zu lesen ist. Der Beitrag von Francesca Prescendi zeigt, wie die aitiologische Geschichte, in deren Mittelpunkt Lucretia steht, als Teil des sozialen Gedächtnisses in der römischen Gesellschaft der politischen Selbstvergewisserung dient. In der zentralen Rolle, die Cicero Clodia, der Schwester des Clodius, in seiner Auseinandersetzung mit diesem politischen Gegner zukommen lässt, zeichnet Rosmarie Günther nach, wie in Rom mit der Verunglimpfung von Frauen Politik betrieben wird - was umgekehrt auf ihre wichtige Funktion in den politischen Strategien der Senatsaristokratie schließen lässt. Die Entstehung des römischen Prinzipats verstärkt diese Bedeutung: Angelika Dierichs präsentiert die Bildnisse der Livia, der Gattin des Augustus, aus archäologisch-kunsthistorischer Sicht und ordnet diese öffentliche Präsenz der Livia in ihren Porträts in den politischen Zusammenhang der neuen Herrschaftsform ein. In diskursanalytischer Perspektive untersucht Thomas Späth die Darstellung der Agrippina, Gattin des Claudius und Mutter des Nero, durch den Geschichtsschreiber Tacitus und weist nach, dass die historiographische Darstellung kaum eine Rekonstruktion der realen Person der Agrippina, sehr wohl aber Rückschlüsse auf die Handlungsbereiche von Frauen in den aristokratischen Häusern des römischen Prinzipats zulässt. Den Abschluss dieses Kapitels bildet ein Blick hinaus auf die Peripherie der römischen Welt, auf die Palmyrenische Herrscherin Zenobia, die sich auf Münzen des Jahres 270/71 n. Chr. Zenobia Augusta nannte: Anja Wieber macht mit philologischer Quellenkritik die besondere Sichtweise antiker Geschichtsschreibung auf eine weibliche Herrscherinnenfigur deutlich. Mit den sechs Beiträgen zur Frage von Macht und Politik in frauenund geschlechtergeschichtlicher Perspektive wird in diesem Kapitel die Vielfalt der methodischen Ansätze und der Forschungsmaterialien wohl am besten greifbar; die Diversität der Methoden führt zur Auflösung einer vermeintlich einheitlichen Definition von Macht und Politik und erlaubt eine differenzierte Betrachtung der unterschiedlichen Aspekte dieser Begriffe: Macht wird in den antiken Gesellschaften, wo politische Beziehungen persönliche Beziehungen sind, fassbar nicht nur in den politischen Strukturen, sondern in den vielfältigen Beziehungen außerhalb, innerhalb und zwischen den Häusern.

Auf die ökonomische Organisation des Haushalts und die Arbeitswelt von Frauen innerhalb und außerhalb des Hauses geht das füfte Kapitel ein. Beate Wagner-Hasel geht den Mythen der geschlechtsspezifischen Arbeitsteilung nach 
und thematisiert die kommunikativen Aspekte weiblicher Arbeit, die Bedeutung von Geweben als textile Medien sowie die weibliche Arbeitsgeselligkeit. Dieser gesellige Aspekt wird auch im Beitrag von Wolfgang Christian Schneider aufgegriffen, der anhand der Schriften des Herondas das Geschäftsgebaren von Frauen in hellenistischer Zeit analysiert. Grundlage für die Ermittlung der Arbeitswelt römischer Frauen bilden vor allem inschriftliche Quellen, deren Auswertung Rosmarie Günther in ihrem Beitrag in den Mittelpunkt stellt. In ihrer Untersuchung kommen vor allem hierarchische Aspekte zur Sprache, die Unterschiede zwischen den Tätigkeitsfeldern römischer Matronen und ihrer Sklavinnen und Freigelassenen, und wird die komplexe Organisationsstruktur eines ranghohen römischen Hauswesens sichtbar. Mehr als in anderen Kapiteln geht es bei der Untersuchung der Arbeitswelt darum, antike Texte (literarische oder philosophische Schriften bzw. Grabinschriften) für die Ermittlung von Sachverhalten auszuwerten, über die zu informieren die Autoren oder Auftraggeber nicht intendiert hatten.

Um Fragen des Verhältnisses von Wirklichkeit, Darstellung und Rezeption geht es auch im letzten Kapitel des Bandes. Erotik und Sexualität werden in einem ersten Beitrag am Beispiel der Hetären thematisiert, die Simone de Beauvoir und anderen sozialistischen Autoren als Prototypus der gebildeten intellektuellen Frau galten, in den jüngeren Arbeiten hingegen als Muster der unterdrückten Frau dargestellt werden. Elke Hartmann zeigt, dass von der Hetäre nicht gesprochen werden kann; sie unterscheidet die gebildete Gefährtin von der Musikerin oder Tänzerin und von der Bordellprostituierten, zwischen denen die Grenzen fliessend sein können, die aber in unterschiedliche gesellschaftliche Kontexte gehören. Angelika Dierichs präsentiert erotische Darstellungen der römischen Kultur und macht darauf aufmerksam, wie wichtig der jeweilige Kontext der Darstellung für seine Interpretation ist. Während von bildlichen Darstellungen mit Sicherheit nicht direkt auf die sexuellen Praktiken geschlossen werden kann, weist die Häufigkeit erotischer Bilder sicher auf eine selbstverständliche Präsenz erotisch konnotierter Körperlichkeit hin, deren Infragestellung durch die Kirchenväter der Beitrag von Wolfgang Christian Schneider aufgreift. Konfliktfrei allerdings spielte sich die Propagierung eines neuen Askese-Ideals nicht ab: Diemut Zittel weist nach, wie sich die christliche Askese in Widerspruch zur traditionellen Definition von Weiblichkeit im Rahmen der Familie stellt.

Damit schließt die Thematik des sechsten Kapitels in gewissem Sinn den Kreis zu den Fragestellungen der Heirat und Zugehörigkeit. Das allerdings bedeutet nicht, dass der vorliegende Band antike Frauen- und Geschlechtergeschichte umfassend behandeln wollte - manche wichtige Fragestellungen bleiben ausgespart. Die in der aktuellen Forschung wichtigen Arbeiten zur Konzeptualisierung des Körpers ${ }^{58}$ oder zur weiblichen und männlichen Homoerotik ${ }^{59}$ konnten nicht auf-

58 Vgl. oben, Anm. 18. 
gegriffen werden; nicht thematisiert wird das Christentum in seiner institutionellen Dimension, etwa die Rolle von Frauen in der Ämterhierarchie. ${ }^{60}$ Auch die neuen Forschungen zur Konstruktion von Männlichkeit und Weiblichkeit kommen nur in Ansätzen zur Sprache. ${ }^{61}$ Doch es wäre auch das exakte Gegenteil der Absicht des vorliegenden Bandes, eine abschließende Sicht auf die Frauen- und Geschlechtergeschichte der Antike vorzulegen. Er will vielmehr einladen zum Weiterdenken und zur Entdeckung der Alten Geschichte mit neuen Fragen.

59 Für die europäische Forschung ein wichtiger Ausgangspunkt war FoucAults Histoire de la sexualité (vgl. vor allem die beiden 1984 erschienenen Bände zur Antike); Hinweise auf neuere Arbeiten finden sich oben in Anm. 22. Vgl. auch den Forschungsbericht WALDNER, Sexualität zwischen Reinheit und Befleckung 1998.

60 EISEN, Amtsträgerinnen im frühen Christentum 1996.

61 Vgl. oben, Anm. 20. 